\title{
The Use of Delay-Locked Loop Signals in DS/CDMA Receiver for Multiple-Access Interference Reduction
}

\author{
Subramaniam Thayaparan, Tung-Sang Ng, Fellow, IEEE, and Jiangzhou Wang, Senior Member, IEEE
}

\begin{abstract}
This paper presents a coherent demodulation scheme that reduces the multiple-access interference (MAI) considerably in a band-limited direct-sequence code-division multiple-access system. The despreading signal is obtained from a combination of the conventional despreading signal and its weighted early and late versions that are used in the delay-locked loop (DLL). It is shown that with appropriate choice of the weighting parameter of the early-late signals of the DLL, the signal-to-interference-plus-noise ratio of the decision variable is increased, which leads to improvement in the bit-error performance and hence the potential increase in the system capacity by about $25 \%$. The analyses are performed in the frequency domain. The effect of imperfect synchronization on the system performance is also analyzed.
\end{abstract}

Index Terms-CDMA capacity, code-division multiple-access (CDMA) interference mitigation, delay-locked loop, multipleaccess interference, spread-spectrum receiver.

\section{INTRODUCTION}

$\mathbf{T}$ HE major limitation to the capacity of a direct-sequence (DS) spread-spectrum (SS) code-division multiple-access (CDMA) system is due to the multiple-access interference (MAI). MAI suppression schemes previously proposed include multiuser detection [1]-[5] and noise whitening techniques [6], [7]. The implementation of these techniques requires either wider system bandwidth or explicit knowledge of the estimates of interference parameters. Recently, specially designed adjustable despreading waveform, whereas the spreading chip waveform is the conventional, has been proposed [8]-[11] for DS/CDMA systems. The purpose is to optimize the shape of the chip waveform in the receiver to reduce MAI and to increase the system capacity. A major advantage of such method is that it does not require explicit knowledge of interference parameters, and the choice of the optimal value for the single adjustable parameter depends only on the signal-to-interference-plus-noise ratio (SINR). In practical implementation, the optimal parameter (or an approximate value of it) can be precomputed off-line. The capacity increase proposed in previous papers [8]-[11]

Manuscript received March 28, 2004; revised September 22, 2004, January 26, 2005, August 19, 2005, and August 31, 2005. This work was supported by the Hong Kong Research Grants Council (RGC) of The Hong Kong Government and the CRCG of the University of Hong Kong. The review of this paper was coordinated by Dr. Z. Xu.

S. Thayaparan is with Agilent Technologies, 768923, Singapore (e-mail: sthay@ singnet.com.sg).

T. S. Ng is with the Department of Elecrical and Electronic Engineering, University of Hong Kong, Hong Kong (e-mail: tsng@eee.hku.hk).

J. Wang is with the Department of Electronics, University of Kent, Kent CT2 7NZ, U.K. (e-mail: J.Z.Wang@kent.ac.uk).

Digital Object Identifier 10.1109/TVT.2006.874575 is obtained with a considerable increase in receiver circuit complexity to generate the specially designed chip waveforms. This paper suggests a scheme that considerably reduces this circuit complexity by utilizing the early and late pseudo-noise (PN) signals that are already available in a CDMA receiver.

In this paper, we proposed a coherent demodulation scheme for band-limited DS/CDMA systems that has the additional property of reducing the MAI. In this scheme, the despreading signal is obtained from a linear combination of the conventional despreading signal and its weighted early and late versions, which are used in the delay-locked tracking loop. It will be shown in this paper that for a fixed early-late spacing of the delay-locked loop (DLL), the SINR of the decision variable can be improved by an appropriate choice of the weighting parameter for the early-late signals. Although variation of the early-late spacing of the DLL can also affect the system performance, in a practical implementation, it is not desirable to continuously adjust the early-late spacing of the DLL. Our focus is therefore on the choice of the weighting parameter for the early-late signals.

In a DLL, it is unavoidable that tracking errors exist. Large tracking error on the DLL degrades the system performance. We shall illustrate, using a practical example, that with a tracking error as high as 0.1 chip time, the proposed scheme still can improve the SINR by about $1 \mathrm{~dB}$, which can be translated into a $25 \%$ increase in capacity.

The rest of the paper is organized as follows: In Section II, the system model is described. Section III presents the derivation of SINR of the decision variable for band-limited DS/CDMA system and its optimization with respect to different system parameters. Numerical examples, the effect of synchronization error on performance and the capacity of the systems are analyzed in Section IV. Finally, conclusions are given in Section V.

\section{System Model}

\section{A. Transmitter Model}

We consider a DS/CDMA system having $K$ active users sharing the same spectrum. Fig. 1 shows the transmitter for the $i$ th user. The modulation type considered is binary phase shift keying (BPSK) with band-limited rectangular chip spreading waveform. The data signal $d_{i}(t)$ and the spreading signal $c_{i}(t)$ for the $i$ th user are given by

$$
d_{i}(t)=\sum_{j=-\infty}^{\infty} d_{i}^{j} p_{d}\left(t-j T_{d}\right)
$$




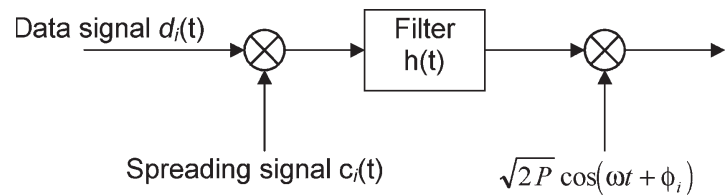

Fig. 1. Block diagram of the transmitter for the $i$ th user.

and

$$
c_{i}(t)=\sum_{j=-\infty}^{\infty} c_{i}^{j} p_{c}\left(t-j T_{c}\right)
$$

where $T_{c}$ is the chip duration, $T_{d}$ is the data bit duration, the data bit waveform $p_{d}(t)=1$ for $0 \leq t<T_{d}$ and $p_{d}(t)=0$ elsewhere, and the chip waveform $p_{c}(t)=1$ for $0 \leq t<T_{c}$ and $p_{c}(t)=0$ elsewhere. The spreading sequence is assumed to be long and periodic with period much larger than $N$, where $N$ represents $T_{d} / T_{c}$. Therefore, we model $c_{i}^{j}$ and $d_{i}^{j}$ as independent random variables having values +1 or -1 with equal probabilities. Filtering must be used to eliminate the out-of-band radiation. Therefore, the product of $c_{i}(t) d_{i}(t)$ is band-limited using a low-pass filter that has a transfer function $H(f)$ before transmission. Thus, the transmitted signal for the $i$ th user is

$$
s_{i}(t)=\sqrt{2 P_{i}}\left\{b_{i}(t) \otimes h(t)\right\} \cos \left(\omega t+\phi_{i}\right)
$$

where $P_{i}$ is the transmitted power for the $i$ th user, $b_{i}(t)=$ $c_{i}(t) d_{i}(t), h(t)$ is the impulse response of the filter $H(f), \omega$ is the carrier frequency, $\phi_{i}$ is the random phase of the carrier signal, and the notation $\otimes$ denotes the convolution operator.

\section{B. Conventional Receiver Model}

The received power of all users' signal are assumed to be the same due to the power control in the CDMA system. The received signal for the aforementioned transmitter model can therefore be expressed as

$$
r(t)=\sqrt{2 P} \sum_{i=1}^{K}\left\{b_{i}\left(t-\tau_{i}\right) \otimes h\left(t-\tau_{i}\right)\right\} \cos \left(\omega t+\theta_{i}\right)+n(t)
$$

where $P$ is the received signal power of all users, $\theta_{i}$ and $\tau_{i}$ are the received carrier phase and the time delay of the $i$ th user, respectively, and $n(t)$ is the additive Gaussian white noise with double-sided power spectral density of $N_{o} / 2$. The random phase $\theta_{i}$ is assumed uniformly distributed in $[0,2 \pi]$, whereas $\tau_{i}$ is uniformly distributed over $T_{d}$. A typical $i$ th user receiver structure using the matched filter is shown in Fig. 2. In the conventional receiver, the despreading signal is the locally generated replica of the spreading signal $c_{i}(t)$. For the receiver to be matched, the spreading and the despreading signals are band-limited by the low-pass filters with the same transfer function denoted by $H(f)$ [12].

The noncoherent DLL structure of a typical conventional receiver [13], [14] is also shown in the figure to compare it with the proposed system. In the DLL (Fig. 2), the early and late correlator signals are squared and filtered by a low-pass filter to remove the data modulation effect. The error signal $e(t)$ is the difference of the early and late signals so obtained, and it is fed to the loop filter. The output of the loop filter drives the PN code generator that corrects the code phase error $\varepsilon$. The detailed analysis for the performance of the DLL, in terms of root-mean-square (rms) tracking error and the mean time to lose lock, can be found in [13] and [14]. Although we study our system model using the noncoherent DLL, the coherent DLL is also applicable to our study because the proposed scheme uses only the early and late despreading signals from the DLL.

\section{Proposed Receiver Structure}

Fig. 3 shows the block diagram of the proposed receiver structure. The despreading signal $\hat{c}_{i}(t)$ is obtained by the combinations of the locally generated replica of the spreading signal $c_{i}(t)$ and its early and late versions $c_{i}(t-\Delta)$ and $c_{i}(t+\Delta)$. The weightings to the early and late signals are chosen to be identical, so that the cross-correlation function of the spreading and despreading signals is symmetrical. In addition, equal weighting of the early and late signals will also result in tracking errors having symmetrical effects on performance. It follows from Fig. 3 that the resultant despreading signal $\hat{c}_{i}(t)$ of the $i$ th user in the proposed scheme is

$$
\hat{c}_{i}(t)=c_{i}(t)-\alpha\left\{c_{i}(t-\Delta)+c_{i}(t+\Delta)\right\} .
$$

The parameter $\alpha$ is to be adjusted to optimize the system performance. The range of $\alpha$ is $0 \leq \alpha \leq 1 / 2$, and the details will be given in Section III-B. Note that the proposed demodulation scheme is conventional when the parameter $\alpha=0$. The structure of the DLL in the proposed scheme is identical to that of the conventional system. Hence the early-late spacing of the DLL is $2 \Delta$. The despreading sequence in (5) contains not only the early and late versions of the PN sequence but also the current version of the PN sequence. It is also possible to include more assistant spreading signals in (5). However, the additional SINR gain obtained by introducing more assistant spreading signals will be negligible compared with the increase in the system complexity. The despreading signal (5) is constructed in a way to suppress more MAI than that of the conventional DLL loop. The generation of this despreading signal (5) increases the system hardware complexity compared with that of conventional demodulation scheme, although it has less complexity compared with the schemes proposed in [8]-[11].

\section{ANALYSis AND OptimizATION OF RECEIVER SINR}

We consider the first user of interest and analyze the coherent receiver given in Fig. 3. The conditional output of the arbitrary data symbol $d_{1}^{\lambda}$ for the first user is denoted by $\beta_{1}(\lambda)$. The decision variable $\beta_{1}(\lambda)$ after the matched filter can be expressed as

$$
\begin{aligned}
\beta_{1}(\lambda)= & \int_{\lambda T_{d}+\tau_{1}}^{(\lambda+1) T_{d}+\tau_{1}} 2 r(t)\left\{\hat{c}_{1}(t+\varepsilon-\right. \\
& \left.\times \cos \left(\omega t+\hat{\theta}_{1}\right) \otimes h\left(t+\varepsilon-\tau_{1}\right)\right\} \\
&
\end{aligned}
$$

where $\hat{\theta}_{1}$ is the carrier phase estimate at the receiver of the first user, and $\varepsilon$ is the tracking error introduced by the DLL. 


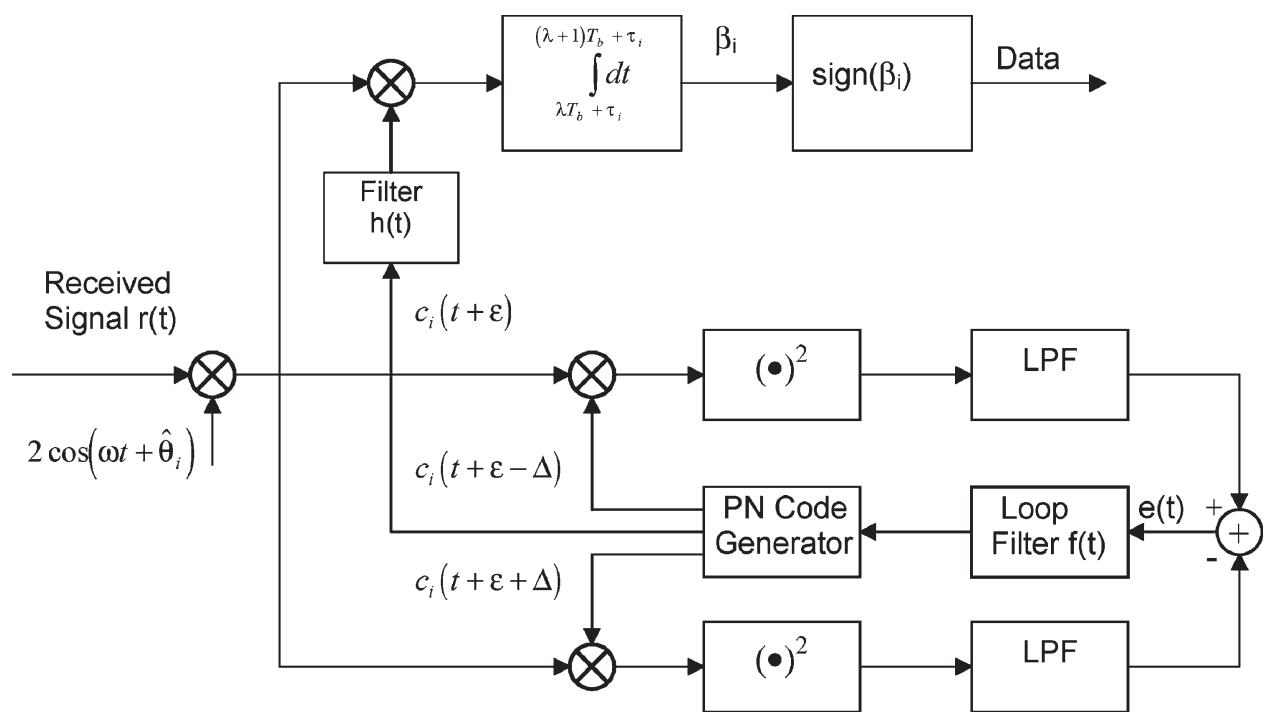

Fig. 2. Block diagram of the conventional receiver for the $i$ th user.

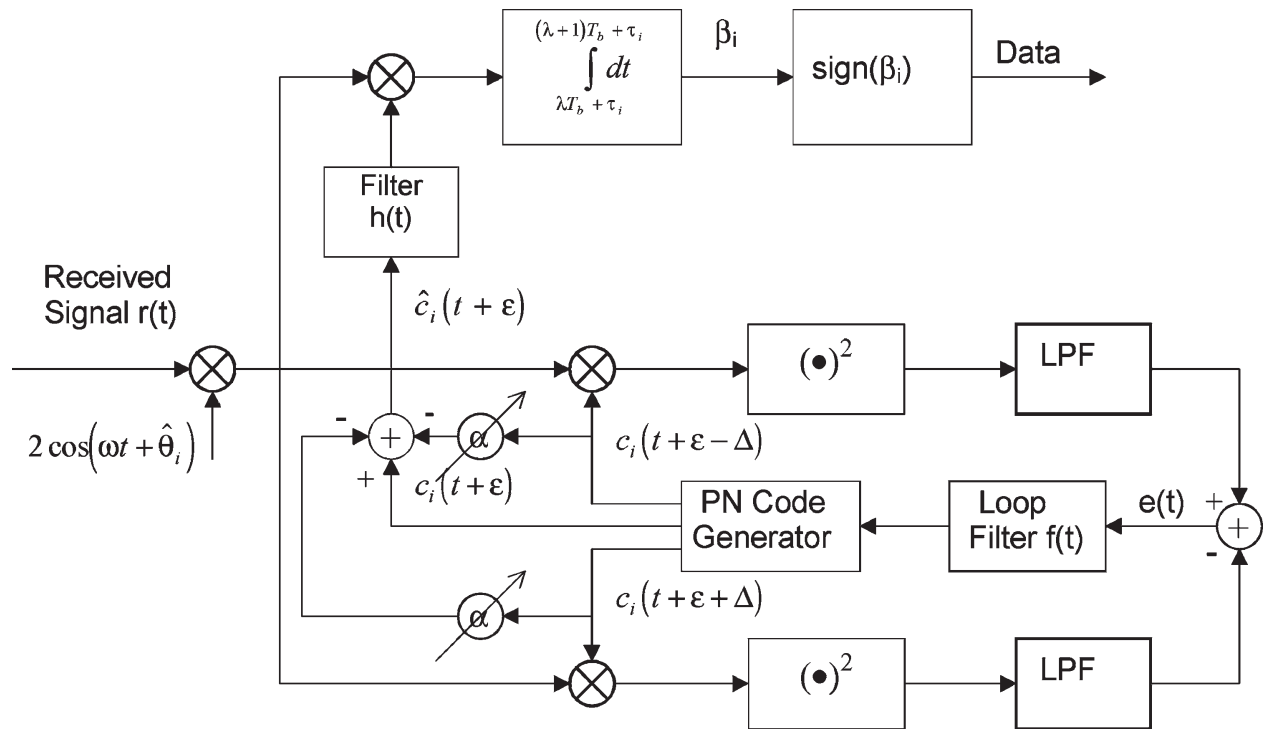

Fig. 3. Block diagram of the proposed receiver for the $i$ th user.

We neglect the error in the estimate of $\hat{\theta}_{1}$ for simplicity of analysis, and therefore, $\theta_{1}=\hat{\theta}_{1}$. In the following, we optimize the system performance by selecting the parameter $\alpha$ for a given early-late time spacing $\Delta$ to maximize the SINR in the decision variable $\beta_{1}(\lambda)$.

\section{A. SINR at the Decision Variable}

As band-limited received signals are considered, it is more convenient to derive the SINR of the decision variable using the frequency-domain approach. Substituting $r(t)$ in (4) into (6), the double frequency terms can be neglected because the data rate is much lower than the carrier frequency. Equation (6) can be separated into three parts as

$$
\beta_{1}(\lambda)=S_{1}^{\lambda}(\alpha, \Delta)+N_{1}^{\lambda}(\alpha, \Delta)+\sum_{i=2}^{K} I_{i}^{\lambda}(\alpha, \Delta)
$$

where $S_{1}^{\lambda}(\alpha, \Delta)$ is the desired signal component, $N_{1}^{\lambda}(\alpha, \Delta)$ is the white noise component, and $\sum_{i=2}^{K} I_{i}^{\lambda}(\alpha, \Delta)$ is the MAI components. The desired signal component $S_{1}^{\lambda}(\alpha, \Delta)$ can be extracted from (6) as follows:

$$
\begin{aligned}
S_{1}^{\lambda}(\alpha, \Delta)= & \sqrt{2 P} \int_{0}^{T_{d}}\left\{c_{1}(t) \otimes h(t)\right\}\left\{\hat{c}_{1}(t+\varepsilon) \otimes h(t+\varepsilon)\right\} d t \\
= & \sqrt{2 P} \int_{-\infty}^{\infty} \int_{-\infty}^{\infty} h(x) h(y) \\
& \times\left\{\int_{0}^{T_{d}} c_{1}(t-x) \hat{c}_{1}(t+\varepsilon-y) d t\right\} d x d y
\end{aligned}
$$


Following the frequency domain analysis as explained in [12], (8) can be further reduced to

$$
\begin{aligned}
S_{1}^{\lambda}(\alpha, \Delta) & =\sqrt{2 P} T_{d} \int_{-\infty}^{\infty} S_{\mathrm{c} \hat{\mathrm{c}}}(f, \alpha, \Delta) e^{j 2 \pi f \varepsilon}|H(f)|^{2} d f \\
& =2 \sqrt{2 P} T_{d} \int_{0}^{\infty} S_{\mathrm{c} \hat{\mathrm{c}}}(f, \alpha, \Delta) \cos (2 \pi f \varepsilon)|H(f)|^{2} d f
\end{aligned}
$$

where $S_{\mathrm{c} \hat{\mathrm{c}}}(f, \alpha, \Delta)$ is the cross-power spectral density of spreading and despreading signals $c_{1}(t)$ and $\hat{c}_{1}(t)$. From (6), the white noise component $N_{1}^{\lambda}(\alpha, \Delta)$ can be expressed as

$$
N_{1}^{\lambda}(\alpha, \Delta)=\int_{0}^{T_{d}} 2 n(t)\left\{\hat{c}_{1}(t+\varepsilon) \otimes h(t+\varepsilon)\right\} \cos \left(\omega t+\hat{\theta}_{1}\right) d t .
$$

Following the approach in [12], the variance of the white noise term $N_{1}^{\lambda}(\alpha, \Delta)$, denoted by $\sigma_{N}^{2}(\alpha, \Delta)$, can be obtained as

$$
\sigma_{N}^{2}(\alpha, \Delta)=N_{o} T_{d} \int_{-\infty}^{\infty} S_{\hat{\mathrm{c}} \hat{\mathrm{c}}}(f, \alpha, \Delta)|H(f)|^{2} d f
$$

where $S_{\hat{c} \hat{c}}(f, \alpha, \Delta)$ is the power spectral density of the despreading signal $\hat{c}_{1}(t)$. The $i$ th couser causes an interference $I_{i}^{\lambda}(\alpha, \Delta)$, which is given by

$$
\begin{aligned}
I_{i}^{\lambda}(\alpha, \Delta)=\sqrt{2 P} \cos \left(\theta_{i}-\hat{\theta}_{1}\right) & \int_{0}^{T_{d}}\left\{d_{i}\left(t-\tau_{i}\right) c_{i}\left(t-\tau_{i}\right) \otimes h\left(t-\tau_{i}\right)\right\} \\
& \times\left\{\hat{c}_{1}(t+\varepsilon) \otimes h(t+\varepsilon)\right\} d t . \quad \text { (12) }
\end{aligned}
$$

Next, we estimate the variance of the MAI term $\sum_{i=2}^{K} I_{i}^{\lambda}(\alpha, \Delta)$, denoted by $\sigma_{I}^{2}(\alpha, \Delta)$, by following the same approach given in [12]. Inasmuch as the phase delay $\theta_{i}-\hat{\theta}_{1}$ is a uniform $[0,2 \pi]$ independent random variable and independent to the time delay $\tau_{i}$, the components of $\sum_{i=2}^{K} I_{i}^{\lambda}(\alpha, \Delta)$ are not correlated among each other. It follows that the variance $\sigma_{I}^{2}(\alpha, \Delta)$ can be expressed as $P \sum_{i=2}^{K} \sigma_{i}^{2}(\alpha, \Delta)$, where $\sigma_{i}^{2}(\alpha, \Delta)$ is the variance of the integral term in (12). By treating $I_{i}^{\lambda}(\alpha, \Delta)$ as an integral of a wide-sense stationary process, the variance $\sigma_{i}^{2}(\alpha, \Delta)$ becomes [12]

$$
\begin{aligned}
\sigma_{i}^{2}(\alpha, \Delta)=T_{d} \int_{-T_{d}}^{T_{d}}(1 & \left.+\frac{|\tau|}{T_{d}}\right)\left\{R_{c_{i} c_{i}}(\tau) \otimes h(\tau) \otimes h(-\tau)\right\} \\
& \times\left\{R_{\hat{\mathrm{c}} \hat{\mathrm{c}}}(\tau) \otimes h(\tau) \otimes h(-\tau)\right\} d \tau
\end{aligned}
$$

where $R_{c_{i} c_{i}}(\tau)$ and $R_{\hat{c} \hat{c}}(\tau)$ are the autocorrelation functions of $c_{i}(t)$ and $\hat{c}_{1}(t)$, respectively. In deriving (13), the effect of the couser data sequence $d_{i}(t)$ is neglected because its autocorrelation is much broader than that of couser's spreading sequence. Also in (13), the term $1+\left(|\tau| / T_{d}\right)$ can be approximated to 1 , and the limits of the integral can be extended from $\pm T_{d}$ to $\pm \infty$ because of two reasons, i.e., 1) $T_{c} \ll T_{d}$ and 2) the autocorrelation functions $R_{c_{i} c_{i}}(\tau)$ and $R_{\hat{\mathrm{c}} \hat{\mathrm{c}}}(\tau)$ are negligible when $|\tau|>T_{c}$. Thus, the integral term in (13) becomes $\left.\left[\left\{S_{c_{i} c_{i}}(f) H(f) H(-f)\right\} \otimes\left\{S_{\hat{\mathrm{c}} \hat{\mathrm{c}}}(f) H(f) H(-f)\right\}\right]\right|_{f=0}$, where $S_{c_{i} c_{i}}(f)$ is the power spectral density of the spreading signal $c_{i}(t)$. Assuming all users' spreading signals have the same power spectral densities, the variance $\sigma_{I}^{2}(\alpha, \Delta)$ of the MAI term $\sum_{i=2}^{K} I_{i}^{\lambda}(\alpha, \Delta)$ is given by

$$
\sigma_{I}^{2}(\alpha, \Delta)=P T_{d}(K-1) \int_{-\infty}^{\infty} S_{\mathrm{cc}}(f) S_{\hat{\mathrm{c}} \hat{\mathrm{c}}}(f, \alpha, \Delta)|H(f)|^{4} d f
$$

where $S_{\mathrm{cc}}(f)$ is the power spectral density of the spreading signal $c_{1}(t)$. Note that the variances $\sigma_{N}^{2}(\alpha, \Delta)$ and $\sigma_{I}^{2}(\alpha, \Delta)$ are independent of the tracking error $\varepsilon$. The power spectral densities $S_{\mathrm{cc}}(f), S_{\mathrm{c} \hat{c}}(f, \alpha, \Delta)$, and $S_{\hat{\mathrm{c}} \hat{c}}(f, \alpha, \Delta)$ are derived in the Appendix. It is now straightforward to obtain the SINR of the decision variable using (9), (11), and (14). From (9) and (11), the signal-to-white-noise power ratio, denoted by $\operatorname{SNR}(\alpha, \Delta)$, is given by

$$
\begin{aligned}
\operatorname{SNR}(\alpha, \Delta) & =\frac{\left\{S_{1}^{\lambda}(\alpha, \Delta)\right\}^{2}}{\sigma_{N}^{2}(\alpha, \Delta)} \\
& =\frac{4 E_{b}}{N_{o}} \frac{\left(\int_{0}^{\infty} S_{\mathrm{c} \hat{c}}(f, \alpha, \Delta) \cos (2 \pi f \varepsilon)|H(f)|^{2} d f\right)^{2}}{\int_{0}^{\infty} S_{\hat{\mathrm{c}} \hat{\mathrm{c}}}(f, \alpha, \Delta)|H(f)|^{2} d f} .
\end{aligned}
$$

Using (9) and (14), the signal-to-MAI power ratio, denoted by $\operatorname{SIR}(\alpha, \Delta)$, is given by

$$
\begin{aligned}
\operatorname{SIR}(\alpha, \Delta) & =\frac{\left\{S_{1}^{\lambda}(\alpha, \Delta)\right\}^{2}}{\sigma_{I}^{2}(\alpha, \Delta)} \\
& =\frac{4 T_{d}}{K-1} \frac{\left(\int_{0}^{\infty} S_{\mathrm{c} \hat{\mathrm{c}}}(f, \alpha, \Delta) \cos (2 \pi f \varepsilon)|H(f)|^{2} d f\right)^{2}}{\int_{0}^{\infty} S_{\mathrm{cc}}(f) S_{\hat{\mathrm{c}} \hat{\mathrm{c}}}(f, \alpha, \Delta)|H(f)|^{4} d f} \\
& =\frac{4 T_{d}}{K-1} \frac{\left(\int_{0}^{\infty} S_{\mathrm{c} \hat{\mathrm{c}}}(f, \alpha, \Delta) \cos (2 \pi f \varepsilon)|H(f)|^{2} d f\right)^{2}}{\int_{0}^{\infty} S_{\mathrm{c} \hat{\mathrm{c}}}^{2}(f, \alpha, \Delta)|H(f)|^{4} d f}
\end{aligned}
$$

Note that in (16), the relationship $S_{\mathrm{c} \hat{c}}^{2}(f, \alpha, \Delta)=$ $S_{\hat{\mathrm{c}} \hat{c}}(f, \alpha, \Delta) S_{\mathrm{cc}}(f)$ following (A5) and (A9) is used. Combining (15) and (16), it follows that

$$
\begin{aligned}
\operatorname{SINR}(\alpha, \Delta) & =\frac{\left\{S_{1}^{\lambda}(\alpha, \Delta)\right\}^{2}}{\sigma_{I}^{2}(\alpha, \Delta)+\sigma_{N}^{2}(\alpha, \Delta)} \\
& =\left[\frac{1}{\operatorname{SNR}(\alpha, \Delta)}+\frac{1}{\operatorname{SIR}(\alpha, \Delta)}\right]^{-1}
\end{aligned}
$$


Hence, the expressions $\operatorname{SNR}(\alpha, \Delta), \quad \operatorname{SIR}(\alpha, \Delta), \quad$ and $\operatorname{SINR}(\alpha, \Delta)$ can be evaluated for a given tracking error $\varepsilon$ and $H(f)$.

\section{B. Optimization of $\operatorname{SINR}(\alpha, \Delta)$}

In this section, we examine $\operatorname{SINR}(\alpha, \Delta)$ with respect to $\alpha$ for a given value of time spacing $\Delta$ and tracking error $\varepsilon$. The expression for $\operatorname{SINR}(\alpha, \Delta)$ in (17) can be expanded using (15) and (16) as shown in (18), shown at the bottom of the page. Referring to the expressions of $S_{\hat{\mathrm{c}} \hat{\mathrm{c}}}(f, \alpha, \Delta)$ and $S_{\mathrm{c} \hat{\mathrm{c}}}(f, \alpha, \Delta)$ in (A5) and (A9), for simplicity of notation, denote

$$
\Theta_{i j}(\varepsilon)=\int_{0}^{\infty} \cos (2 \pi f \varepsilon)\{\cos (2 \pi f \Delta)\}^{i}\left\{S_{\mathrm{cc}}(f)\right\}^{j}|H(f)|^{2 j} d f
$$

which is a function of $\Delta$ and $\varepsilon$. The dependence of $\Delta$ is not explicitly mentioned because it is a constant in a DLL. The subindices $i$ and $j$ are integers that satisfy $0 \leq i$ and $j \leq 2$, respectively. Using $\Theta_{i j}(\varepsilon)$, the expression for $\operatorname{SINR}(\alpha, \Delta)$ can be rewritten as (20), shown at the bottom of the page, where $p=N_{o} / 4 E_{b}$ and $q=K-1 / 4 T_{d}$. For a given $\Delta$, the optimal value of $\alpha$, denoted by $\alpha_{\text {SINR }}^{*}$, that maximizes $\operatorname{SINR}(\alpha, \Delta)$ can be obtained by solving the equation $\partial \operatorname{SINR}(\alpha, \Delta) / \partial \alpha=0$. Following this procedure and after some mathematical manipulations, $\alpha_{\text {SINR }}^{*}$ is obtained as

$$
\begin{aligned}
& \alpha_{\text {SINR }}^{*}=\frac{1}{2} \\
& \quad \times \frac{\Theta_{01}(\varepsilon)\left[p \Theta_{11}(0)+q \Theta_{12}(0)\right]-\Theta_{11}(\varepsilon)\left[p \Theta_{01}(0)+q \Theta_{02}(0)\right]}{\Theta_{01}(\varepsilon)\left[p \Theta_{21}(0)+q \Theta_{22}(0)\right]-\Theta_{11}(\varepsilon)\left[p \Theta_{11}(0)+q \Theta_{12}(0)\right]} .
\end{aligned}
$$

Using (19) and (21), it can be also proven that $0 \leq \alpha_{\text {SINR }}^{*}<0.5$. The $\operatorname{SIR}(\alpha, \Delta)$ can be obtained from (18) as a special case when $E_{b} / N_{o}=\infty$ or from (20) when $p=0$. It is apparent that the values of $\alpha$ that optimizes $\operatorname{SIR}(\alpha, \Delta)$, denoted by $\alpha_{\mathrm{SIR}}^{*}$, can also be obtained from (21) by setting $p=0$. It follows that

$$
\alpha_{\mathrm{SIR}}^{*}=\frac{1}{2} \frac{\Theta_{01}(\varepsilon) \Theta_{12}(0)-\Theta_{11}(\varepsilon) \Theta_{02}(0)}{\Theta_{01}(\varepsilon) \Theta_{22}(0)-\Theta_{11}(\varepsilon) \Theta_{12}(0)} .
$$

It is clear that $\alpha_{\mathrm{SIR}}^{*}$ is independent of $q$ and hence the active number of users $K$. It depends on the system parameters $\Delta$ and $H(f)$ on which $\Theta_{i j}(\varepsilon)$ in (19) depends. For a fixed $\Delta$ and $H(f), \alpha_{\mathrm{SIR}}^{*}$ is a constant. The optimal $\alpha$ that maximizes $\operatorname{SNR}(\alpha, \Delta)$, denoted by $\alpha_{\mathrm{SNR}}^{*}$, can also be obtained from (21) by setting $q=0$ [equivalent to setting the number of active users $K=1$ in (18)], i.e.,

$$
\alpha_{\mathrm{SNR}}^{*}=\frac{1}{2} \frac{\Theta_{01}(\varepsilon) \Theta_{11}(0)-\Theta_{11}(\varepsilon) \Theta_{01}(0)}{\Theta_{01}(\varepsilon) \Theta_{21}(0)-\Theta_{11}(\varepsilon) \Theta_{11}(0)} .
$$

Consider the case when the tracking error $\varepsilon=0$, which all tracking loops are trying to achieve. It follows that $\alpha_{\mathrm{SNR}}^{*}=0$, which yields the conventional decision variables. This implies that the conventional decision variable actually maximizes the SNR. The substitution of $\varepsilon=0$ in (22) would yield an $\alpha$ that maximizes the SIR of the decision variable. It implies that the proposed receiver is able to reduce the effect of MAI on the decision variable. However, the nonzero value of $\alpha$ decreases the SNR of the decision variable. The tradeoff between the increase in SIR and the decrease in SNR leads to different values of $\alpha_{\text {SINR }}^{*}$ for different $E_{b} / N_{o}$ and different number of users $K$ in the system. We shall explore these relationships in the next section using specific examples.

\section{Numerical Results}

The filters that limit the bandwidth of the system both in the transmitter and the receiver are assumed to be ideal low-pass filters with one-sided bandwidth $B$, i.e.,

$$
H(f)= \begin{cases}1, & |f| \leq B \\ 0, & \text { elsewhere }\end{cases}
$$

The system is therefore band-limited to $2 B$. Although such ideal filters cannot be easily implemented in practice, it serves the purpose of demonstrating the band-limitation effect in this study. In the following, unless specified otherwise, the values for the processing gain $N=255$, the total numbers of active users $K=80$, and the bandwidth $B=1 / T_{c}$ are assumed. Inasmuch as one chip time early-late spacing $\left(\Delta=1 / 2 T_{c}\right)$ is commonly used in the DLL, this value is used as a reference when plotting results.

$$
\operatorname{SINR}(\alpha, \Delta)=\frac{\left(\int_{0}^{\infty} S_{\mathrm{c} \hat{\mathrm{c}}}(f, \alpha, \Delta) \cos (2 \pi f \varepsilon)|H(f)|^{2} d f\right)^{2}}{\frac{N_{o}}{4 E_{b}} \int_{0}^{\infty} S_{\hat{\mathrm{c}} \hat{\mathrm{c}}}(f, \alpha, \Delta)|H(f)|^{2} d f+\frac{K-1}{4 T_{d}} \int_{0}^{\infty} S_{\mathrm{c} \hat{\mathrm{c}}}^{2}(f, \alpha, \Delta)|H(f)|^{4} d f}
$$

$$
\operatorname{SINR}(\alpha, \Delta)=\frac{\left[\Theta_{01}(\varepsilon)-2 \alpha \Theta_{11}(\varepsilon)\right]^{2}}{\left[p \Theta_{01}(0)+q \Theta_{02}(0)\right]-4 \alpha\left[p \Theta_{11}(0)+q \Theta_{12}(0)\right]+4 \alpha^{2}\left[p \Theta_{21}(0)+q \Theta_{22}(0)\right]}
$$




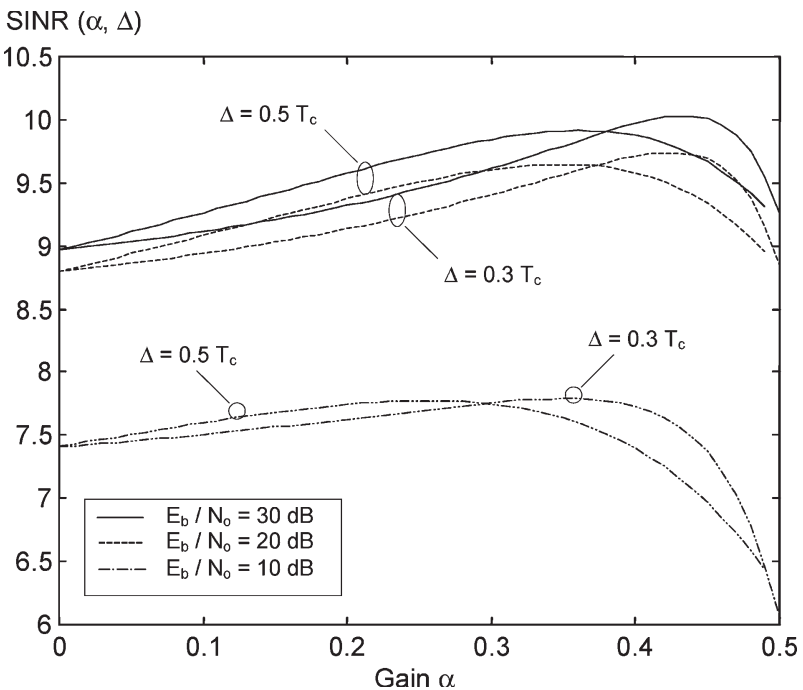

Fig. 4. Plots of $\operatorname{SINR}(\alpha, \Delta)$ against the gain $\alpha$ for $N=255, K=80$, and $B=1 / T_{c}$.

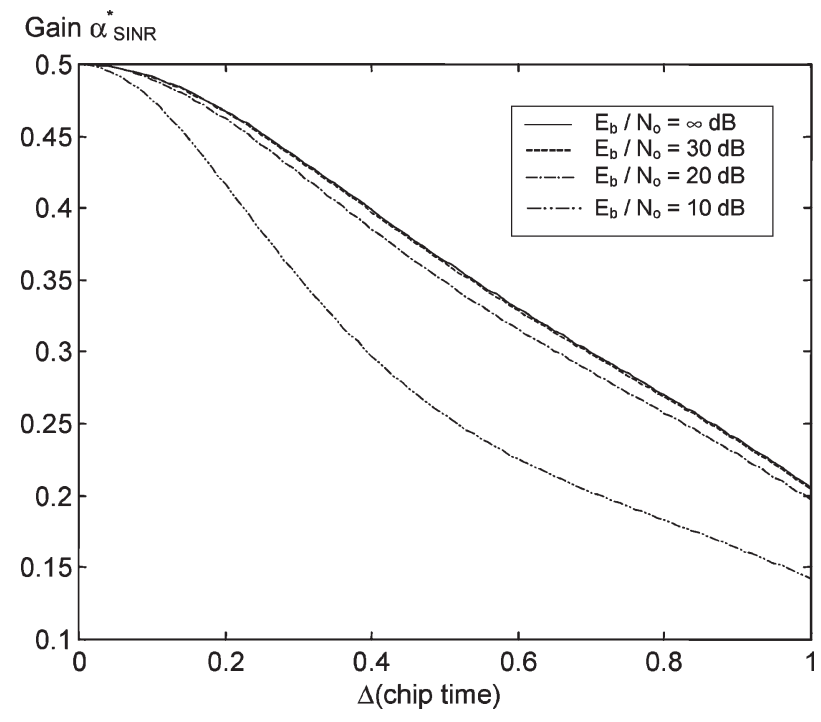

Fig. 5. Plots of optimum $\alpha_{\text {SINR }}^{*}$ against $\Delta$ for $N=255, K=80$, and $B=1 / T_{c}$.

\section{A. Decision Variable $\operatorname{SINR}(\alpha, \Delta)$}

First, the zero tracking error case $(\varepsilon=0)$ is studied. The plots of $\operatorname{SINR}(\alpha, \Delta)$ against $\alpha$ are given in Fig. 4 for $E_{b} / N_{o}$ equals to 10,20 , and $30 \mathrm{~dB}$ and for $\Delta$ equals to $0.3 T_{c}$ and $0.5 T_{c}$. It is clear from the plots that $\operatorname{SINR}(\alpha, \Delta)$ increases when $\alpha$ increases from zero (the case of a conventional demodulation scheme), and there is an optimal value $\alpha_{\text {SINR }}^{*}$ in which $\operatorname{SINR}(\alpha, \Delta)$ achieves its maximum value for a given $E_{b} / N_{o}$ and $\Delta$. These plots also illustrate that $\operatorname{SINR}\left(\alpha_{\text {SINR }}^{*}, \Delta\right)$ can be slightly increased by reducing $\Delta$. Note that the gain in the $\operatorname{SINR}$ given by $\left\{\operatorname{SINR}\left(\alpha_{\text {SINR }}^{*}, \Delta\right)-\operatorname{SINR}(0, \Delta)\right\}$ decreases when $E_{b} / N_{o}$ reduces can be explained by the relatively larger contribution of white noise power in the SINR.

The effect of $\Delta$ on $\alpha_{\text {SINR }}^{*}$ for a given $E_{b} / N_{o}$ is investigated next. Fig. 5 shows $\alpha_{\text {SINR }}^{*}$ in (21) that maximizes $\operatorname{SINR}(\alpha, \Delta)$ against the time spacing $\Delta$ for $E_{b} / N_{o}$ equals $10,20,30$ and $\infty \mathrm{dB}$. Note that the special case $E_{b} / N_{o}=\infty$ is equivalent

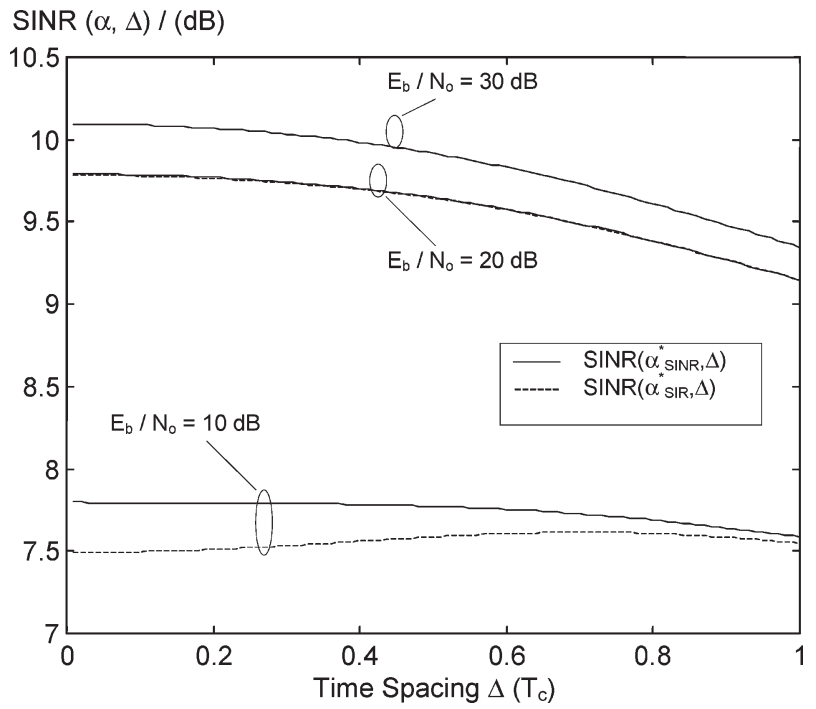

Fig. 6. Plots of $\operatorname{SINR}(\alpha, \Delta)$ against $\Delta$ for 1) $\alpha_{\text {SINR }}^{*}$ and 2) $\alpha_{\text {SIR }}^{*}$.

to the case $p=0$ in (21), and therefore, $\alpha_{\mathrm{SINR}}^{*}=\alpha_{\mathrm{SIR}}^{*} \max$ imizes $\operatorname{SIR}(\alpha, \Delta)$. The value of $\alpha_{\mathrm{SINR}}^{*}$ approaches to $\alpha_{\mathrm{SIR}}^{*}$ when $E_{b} / N_{o}$ increases. For high $E_{b} / N_{o}$, which indicates that contribution from the white noise term is not significant, e.g., $>20 \mathrm{~dB}, \alpha_{\text {SINR }}^{*}$ is approximately constant, i.e., $\alpha_{\text {SINR }}^{*} \cong \alpha_{\text {SIR }}^{*}$ for a given $\Delta$. It is apparent that close to optimum performance can be obtained with a fixed $\alpha$ for high $E_{b} / N_{o}$ situations. However for low $E_{b} / N_{o}$, e.g., $10 \mathrm{~dB}, \alpha_{\text {SINR }}^{*}$ has a considerable difference with $\alpha_{\text {SIR }}^{*}$ due to the influence of white noise power in SINR, and therefore, optimum performance requires a variable $\alpha_{\text {SINR }}^{*}$.

The maximum $\operatorname{SINR}(\alpha, \Delta)$ for each $\Delta$ can be obtained from (20) by substituting the value of $\alpha_{\text {SINR }}^{*}$ in (21). $\operatorname{SINR}\left(\alpha_{\text {SINR }}^{*}, \Delta\right)$ and $\operatorname{SINR}\left(\alpha_{\text {SIR }}^{*}, \Delta\right)$ are plotted against $\Delta$ in Fig. 6 for $E_{b} / N_{o}$ values of 10, 20, and $30 \mathrm{~dB}$. We have the following observation. First, $\operatorname{SINR}\left(\alpha_{\mathrm{SINR}}^{*}, \Delta\right)$ decreases with increasing $\Delta$. However, the change is small for $\Delta \leq 0.5$. Second, from Figs. 5 and 6 , the optimal set of $(\alpha, \Delta)$, which maximizes $\operatorname{SINR}(\alpha, \Delta)$, approaches $\alpha=0.5$ and $\Delta=0$. It is clear from $\hat{c}_{i}(t)$ given in (5) that demodulation cannot be performed for $\alpha=0.5$ and $\Delta=0$ because $\hat{c}_{i}(t)$ vanishes for this set of values of $\alpha$ and $\Delta$, and hence, joint optimization of $\alpha$ and $\Delta$ is not practical. Third, $\operatorname{SINR}\left(\alpha_{\text {SINR }}^{*}, \Delta\right)$ and $\operatorname{SINR}\left(\alpha_{\text {SIR }}^{*}, \Delta\right)$ values are approximately the same when $E_{b} / N_{o}$ is high, and they have considerable difference for low value of $E_{b} / N_{o}$. This reinforces the earlier observation of stronger influence of white noise power on the decision variable at low SNR.

To further elaborate the effect of $E_{b} / N_{o}$ on $\operatorname{SINR}\left(\alpha_{\text {SINR }}^{*}, \Delta\right)$ and $\operatorname{SINR}\left(\alpha_{\mathrm{SIR}}^{*}, \Delta\right)$, Figs. 7 and 8 plot $\operatorname{SINR}\left(\alpha_{\mathrm{SINR}}^{*}, \Delta\right)$ and $\operatorname{SINR}\left(\alpha_{\mathrm{SIR}}^{*}, \Delta\right)$, respectively, against $E_{b} / N_{o}$ for 1) $\Delta=$ $\left.\left.0.1 T_{c}, 2\right) \Delta=0.3 T_{c}, 3\right) \Delta=0.5 T_{c}$, and 4) the conventional system with $\alpha=0$. The values of $\operatorname{SINR}\left(\alpha_{\text {SINR }}^{*}, \Delta\right)$ and $\operatorname{SINR}\left(\alpha_{\text {SIR }}^{*}, \Delta\right)$ are almost the same for $E_{b} / N_{o}>15 \mathrm{~dB}$. However, from Fig. 8, $\operatorname{SINR}\left(\alpha_{\mathrm{SIR}}^{*}, \Delta\right)<\operatorname{SINR}(0, \Delta)$ for $E_{b} / N_{o}<$ $10 \mathrm{~dB}$, which indicates that the conventional system $(\alpha=0)$ is optimal when $E_{b} / N_{o}<10 \mathrm{~dB}$. This reaffirms the conclusion drawn on the conventional decision variable that maximizes SNR, which is given at the end of the last section. The gain 
$\operatorname{SINR}\left(\alpha^{*} \operatorname{sinR}, \Delta\right) /(\mathrm{dB})$

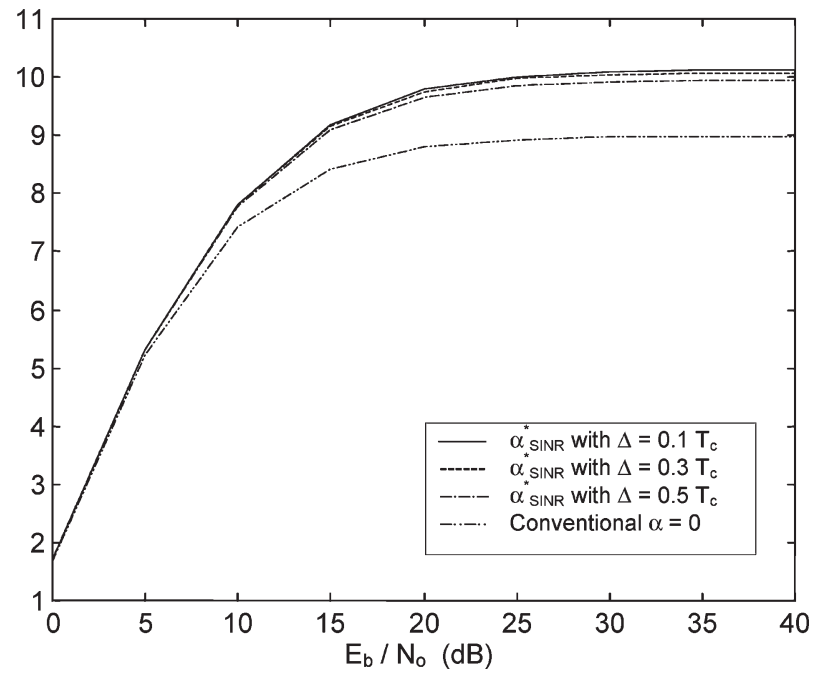

Fig. 7. Plots of $\operatorname{SINR}\left(\alpha_{\text {SINR }}^{*}, \Delta\right)$ against $E_{b} / N_{o}$.

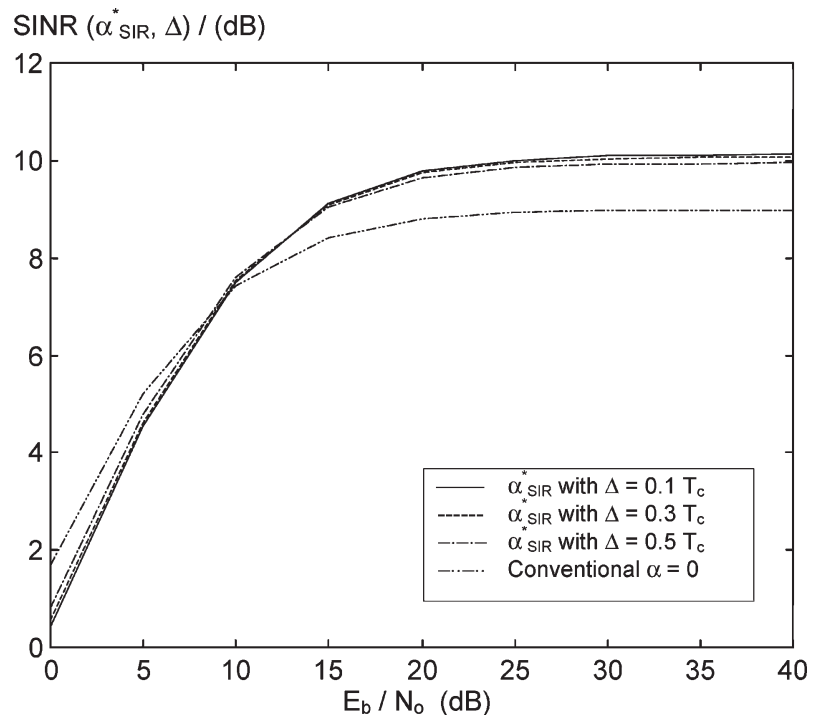

Fig. 8. Plots of $\operatorname{SINR}\left(\alpha_{\mathrm{SIR}}^{*}, \Delta\right)$ against $E_{b} / N_{o}$.

in SINR with $\Delta=0.5 T_{c}$, which is preferred from DLL point of view, is about $1 \mathrm{~dB}$ when compared with the conventional system. It is also clear that reduction in $\Delta$ only results in very small increase in SINR.

It is also imperative to examine the effect of the number of users $K$ on SINR. The plots of $\operatorname{SINR}(\alpha, \Delta)$ against $K$ are shown in Fig. 9 for $E_{b} / N_{o}$ equals to 10,20 , and $30 \mathrm{~dB}$ for the case $\Delta=0.5 T_{c}$. It is obvious that the proposed scheme is more effective in improving the SINR for larger values of $K$ when compared with the conventional system. This illustrates the MAI reduction property of the proposed demodulation scheme and is particularly important when the system is operating near capacity. Fig. 9 also illustrates that the use of $\alpha_{\text {SINR }}^{*}$ optimizes the SINR for any number of active users $K$. The plots of $\operatorname{SINR}(\alpha, \Delta)$ when $\Delta=0.5 T_{c}$ and $E_{b} / N_{o}=10 \mathrm{~dB}$ show that the choice of $\alpha_{\text {SIR }}^{*}$ for $\alpha$ is not an optimized value when the number of active users $K$ is relatively small. In this case, the conventional system $(\alpha=0)$ performs better. This can be explained by the MAI and the white noise power ratio in SINR.

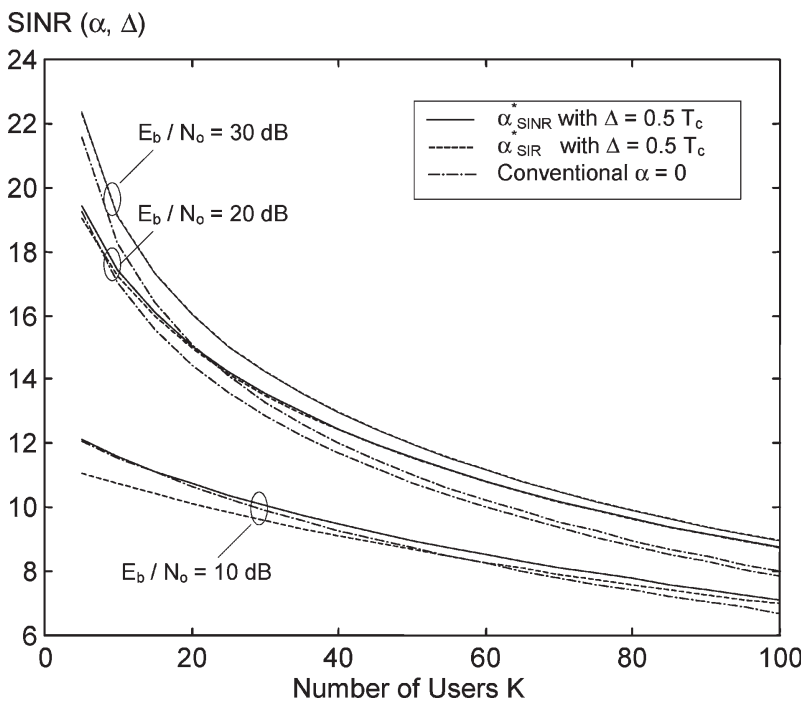

Fig. 9. Plots of $\operatorname{SINR}\left(\alpha_{\text {SIR }}^{*}, \Delta\right)$ against number of users $K$.

\section{B. Effect of Imperfect Synchronization}

In the previous analysis, we have omitted the effect of imperfect synchronization of incoming and locally generated spreading codes. During normal CDMA operations, the tracking error affects the system performance. Note that the tracking error $\varepsilon$ is almost constant for quite a number of data bits because the closed-loop bandwidth of the DLL is much less than the data rate. When the tracking error $\varepsilon$ is present, there will be a reduction in $\operatorname{SINR}\left(\alpha_{\text {SINR }}^{*}, \Delta\right)$. For a given tracking error $\varepsilon$, the SINR gain (in decibels) $G\left(\varepsilon, \alpha_{\text {SINR }}^{*}, \Delta\right)$ of the proposed scheme compared with that of the conventional scheme can be expressed as

$$
\begin{aligned}
G\left(\varepsilon, \alpha_{\text {SINR }}^{*}, \Delta\right)=10 \log _{10} & \left\{\operatorname{SINR}\left(\varepsilon, \alpha_{\text {SINR }}^{*}, \Delta\right)\right\} \\
- & 10 \log _{10}\{\operatorname{SINR}(\varepsilon, 0, \Delta)\} .
\end{aligned}
$$

We study the effect of tracking error on the proposed system with $\Delta=0.1 T_{c}$ and $\Delta=0.5 T_{c}$. Note that the values of $\alpha_{\text {SINR }}^{*}$ in (21) have to be evaluated for each tracking error $\varepsilon$ due to its dependence on $\varepsilon$. Fig. 10 shows the SINR gain $G\left(\varepsilon, \alpha_{\text {SINR }}^{*}, \Delta\right)$ against tracking error $\varepsilon$ for $E_{b} / N_{o}=30 \mathrm{~dB}$. The curves of $G\left(\varepsilon, \alpha_{\text {SINR }}^{*}, \Delta\right)$ in Fig. 10 are evaluated using 1) $\alpha_{\text {SINR }}^{*}$ obtained with $\varepsilon$ and 2) $\alpha_{\text {SINR }}^{*}$ obtained with $\varepsilon=0$. It is obvious that case 1 performs better than case 2 . However case 1 requires feedback of the estimated $\varepsilon$, say from the DLL, to compute $\alpha_{\text {SINR }}^{*}$. On the other hand, $\alpha$ for case 2 is predefined with SINR. In both cases, the SINR gain is reduced with increasing tracking error $\varepsilon$ as expected. It is also clear from Fig. 10 that the proposed scheme results in better SINR of the decision variable for tracking error up to $0.4 T_{c}$ when compared with that of the conventional system. Also note that the SINR gains obtained from using both $\alpha_{\text {SINR }}^{*}$ with $\varepsilon \neq 0$ and with $\varepsilon=0$ are almost the same until the tracking error becomes larger than $0.1 T_{c}$. During normal CDMA operating conditions, the tracking error is usually kept small, and it suffices to use $\alpha_{\text {SINR }}^{*}$ with $\varepsilon=0$ for practical implementation.

When implementing this scheme, a predefined threshold value of $E_{b} / N_{o}$ can be used to select the value of $\alpha$. For 
$\mathrm{G}\left(\varepsilon, \alpha_{\mathrm{SINR}, \Delta)}^{*}\right.$

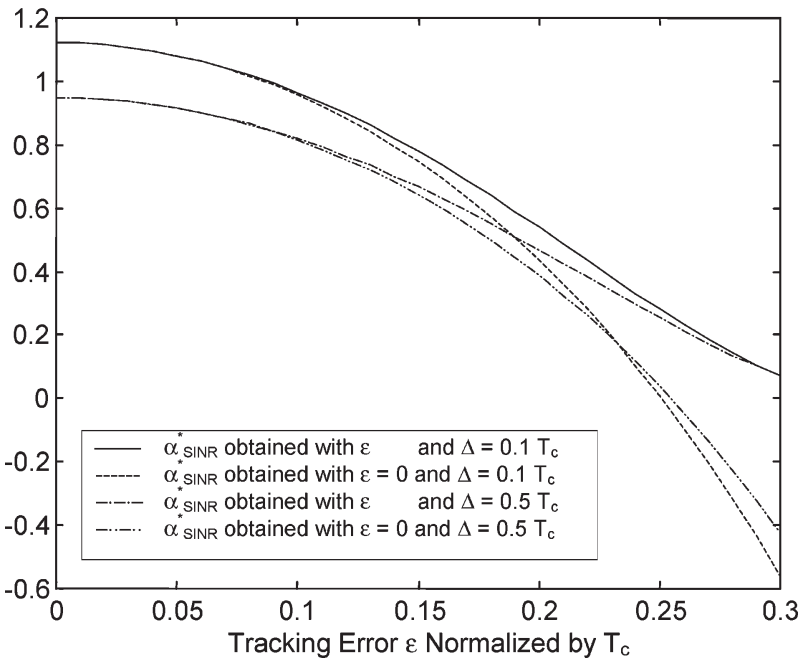

Fig. 10. Plots of $G\left(\varepsilon, \alpha_{\text {SINR }}^{*}, \Delta\right)$ against the tracking error $\varepsilon$ for $E_{b} / N_{o}$ of $30 \mathrm{~dB}$.

example, the conventional system with $\alpha=0$ can be used when $E_{b} / N_{o}$ is below this predefined threshold value of $E_{b} / N_{o}$. Either $\alpha_{\text {SIR }}^{*}$ or $\alpha_{\text {SINR }}^{*}$ can be selected for $\alpha$ when $E_{b} / N_{o}$ is above this threshold. Suppose the proposed scheme is used in a fading channel where $E_{b} / N_{o}$ varies widely, it is possible to have high switching activity while choosing the values for $\alpha$ from $0, \alpha_{\mathrm{SINR}}^{*}$, or $\alpha_{\mathrm{SIR}}^{*}$. However the effect of this switching activity is expected to be minimal because the data rate is much higher than the switching rate of $\alpha$.

\section{System Capacity}

The performance of the CDMA system operating near capacity depends primarily on $\operatorname{SIR}(\alpha, \Delta)$ because the additive white noise power is negligible compared with the MAI noise power. Suppose the proposed and the conventional schemes have the same SIR when the number of active users is $K_{o}$ and $K$, respectively, we can derive (26), shown at the bottom of the page, using the expression for $\operatorname{SIR}(\alpha, \Delta)$ in (16). Using $K$, $K_{o} \gg 1$, and $H(f)$ in (22) with $B=1 / T_{c}$, numerical integration yields the following results. When there is no tracking error $(\varepsilon=0), K_{o}=1.2995 K$ for $\Delta=0.1 T_{c}$ and $K_{o}=1.2474 K$ for $\Delta=0.5 T_{c}$. This means the system capacity is increased by about $30 \%$ by selecting $\Delta=0.1 T_{c}$ and about $25 \%$ by selecting $\Delta=0.5 T_{c}$. Similarly, when the tracking error is $0.1 T_{c}$, the capacity of the proposed system is increased by $25.3 \%$ with $\Delta=0.1 T_{c}$ and about $21 \%$ with $\Delta=0.5 T_{c}$.

For a given set of parameters, the performance presented in [8]-[11] in terms of the capacity increase and SINR gain is slightly higher compared with the values given in this paper. The difference is not significant when comparing the advantage of easy implementation of the proposed scheme in this paper.

\section{CONCLUSION}

In this paper, a demodulation scheme for band-limited DS/CDMA system has been proposed and analyzed. The despreading signal is obtained from combining the conventional despreading signal with an appropriate weighting of the early and late despreading signals of the DLL. It has been shown that the proposed demodulation scheme improves the SINR of the decision variable that leads to an increase in the system capacity. For system operating in high $E_{b} / N_{o}$ and with a fixed $\Delta$, which is normally the case for a CDMA system, numerical examples show that a fixed $\alpha$ can yield near-optimal performance in most conditions. Numerical examples have also shown that with tracking error as high as $0.1 T_{c}$, the proposed scheme can still improve the SINR by $1 \mathrm{~dB}$ when compared with the conventional scheme. This can be translated into about $25 \%$ gain in capacity.

\section{APPENDIX}

We derive the auto- and cross-power spectral densities of the spreading and despreading signals. The power spectral density $S_{\hat{\mathrm{c}} \hat{c}}(f, \alpha, \Delta)$ of the despreading signal $\hat{c}_{1}(t)$ is expressed by the Fourier transform of its autocorrelation function, i.e.,

$$
S_{\hat{\mathrm{c}} \hat{\mathrm{c}}}(f, \alpha, \Delta)=\int_{-\infty}^{\infty} R_{\hat{\mathrm{c}} \hat{\mathrm{c}}}(z, \alpha, \Delta) e^{-2 \pi f z} d z
$$

where $R_{\hat{\mathrm{c}} \hat{\mathrm{c}}}(z, \alpha, \Delta)$ is the autocorrelation function of $\hat{c}_{1}(t)$ given by

$$
R_{\widehat{\mathrm{c}} \hat{\mathrm{c}}}(z, \alpha, \Delta)=\int_{-\infty}^{\infty} \hat{c}_{1}(t) \hat{c}_{1}(t+z) d t .
$$

Combining (5) and (A2), the autocorrelation function $R_{\hat{\mathrm{c}} \hat{\mathrm{c}}}(z, \alpha, \Delta)$ can be expressed as

$$
\begin{array}{r}
R_{\hat{\mathrm{c}} \hat{\mathrm{c}}}(z, \alpha, \Delta)=(1+ \\
\left.+\alpha^{2}\right) R_{\mathrm{cc}}(z)-2 \alpha\left\{R_{\mathrm{cc}}(z+\Delta)+R_{\mathrm{cc}}(z-\Delta)\right\} \\
+\alpha^{2}\left\{R_{\mathrm{cc}}(z+2 \Delta)+R_{\mathrm{cc}}(z-2 \Delta)\right\}
\end{array}
$$

where $R_{\mathrm{cc}}(z)$ is the autocorrelation function of the spreading signal $c_{1}(t)$, and it is given by

$$
R_{\mathrm{cc}}(z)=\int_{-\infty}^{\infty} c_{1}(t) c_{1}(t+z) d t
$$

$$
\frac{K_{o}-1}{K-1}=\frac{\left\{\int_{0}^{\infty} S_{\mathrm{cc}}^{2}(f)|H(f)|^{4} d f\right\}\left\{\int_{0}^{\infty} S_{\mathrm{c} \hat{\mathrm{c}}}\left(f, \alpha_{o}^{*}, \Delta\right) \cos (2 \pi f \varepsilon)|H(f)|^{2} d f\right\}^{2}}{\left\{\int_{0}^{\infty} S_{\mathrm{c} \hat{\mathrm{c}}}^{2}\left(f, \alpha_{o}^{*}, \Delta\right)|H(f)|^{4} d f\right\}\left\{\int_{0}^{\infty} S_{\mathrm{cc}}(f) \cos (2 \pi f \varepsilon)|H(f)|^{2} d f\right\}^{2}}
$$


Using (A3), the power spectral density $S_{\hat{\mathrm{c}} \hat{\mathrm{c}}}(f, \alpha, \Delta)$ of the despreading signal $\hat{c}_{1}(t)$ in (A1) becomes

$$
\begin{aligned}
& S_{\hat{\mathrm{c}} \hat{\mathrm{c}}}(f, \alpha, \Delta)=\{\left(1+2 \alpha^{2}\right)-2 \alpha\left(e^{2 \pi f \Delta}+e^{-2 \pi f \Delta}\right) \\
&\left.+\alpha^{2}\left(e^{4 \pi f \Delta}+e^{-4 \pi f \Delta}\right)\right\} S_{\mathrm{cc}}(f) \\
&=\{1-2 \alpha \cos (2 \pi f \Delta)\}^{2} S_{\mathrm{cc}}(f) .
\end{aligned}
$$

Similarly, the cross-power spectral density $S_{\mathrm{c} \hat{c}}(f, \alpha, \Delta)$ of the despreading signal $\hat{c}_{1}(t)$ and the spreading signal $c_{1}(t)$ is expressed by the Fourier transform of their cross-correlation function, i.e.,

$$
S_{\mathrm{c} \hat{\mathrm{c}}}(f, \alpha, \Delta)=\int_{-\infty}^{\infty} R_{\mathrm{c} \hat{\mathrm{c}}}(z, \alpha, \Delta) e^{-2 \pi f z} d z
$$

where $R_{\mathrm{c} \hat{\mathrm{c}}}(z, \alpha, \Delta)$ is the cross-correlation function of the despreading signal $\hat{c}_{1}(t)$ and the spreading signal $c_{1}(t)$ given by

$$
R_{\mathrm{c} \hat{\mathrm{c}}}(z, \alpha, \Delta)=\int_{-\infty}^{\infty} c_{1}(t) \hat{c}_{1}(t+z) d t .
$$

Using (5) and (A7), the cross-correlation function $R_{\mathrm{c} \hat{\mathrm{c}}}(z, \alpha, \Delta)$ can be expressed as

$$
R_{\mathrm{c} \hat{\mathrm{c}}}(z, \alpha, \Delta)=R_{\mathrm{cc}}(z)-\alpha\left\{R_{\mathrm{cc}}(z+\Delta)+R_{\mathrm{cc}}(z-\Delta)\right\} .
$$

Thus, the cross-power spectral density $S_{\mathrm{cc}}(f, \alpha, \Delta)$ can be obtained by combining (A6) and (A8), i.e.,

$$
\begin{aligned}
S_{\mathrm{c \hat {c }}}(f, \alpha, \Delta) & =\left\{1-\alpha\left(e^{2 \pi f \Delta}+e^{-2 \pi f \Delta}\right)\right\} S_{\mathrm{cc}}(f) \\
& =\{1-2 \alpha \cos (2 \pi f \Delta)\} S_{\mathrm{cc}}(f) .
\end{aligned}
$$

The power spectral density $S_{\mathrm{cc}}(f)$ of the spreading signal $c_{1}(t)$ is well known, and it can be easily obtained by $S_{\mathrm{cc}}(f)=T_{c} \operatorname{sinc}^{2}\left(\pi f T_{c}\right)$.

\section{REFERENCES}

[1] Z. Xie, R. T. Short, and C. K. Rushforth, "A family of suboptimum detectors for coherent multiuser communications," IEEE J. Sel. Areas Commun., vol. 8, no. 4, pp. 683-690, May 1990.

[2] M. Varanasi and B. Aazhang, "Multistage detection in asynchronous code-division multiple access communications," IEEE Trans. Commun., vol. 38, no. 4, pp. 509-519, Apr. 1990.

[3] _ - "Near-optimum detection in synchronous code-division multiple access communications," IEEE Trans. Commun., vol. 39, no. 5, pp. 725736, May 1991.

[4] A. Duel-Hallen, "Decorrelating decision-feedback multiuser detector for synchronous code-division multiple access channels," IEEE Trans. Commun., vol. 41, no. 2, pp. 285-290, Feb. 1993.

[5] Y. C. Yoon and H. L. Leib, "Matched filters with interference suppression capabilities for DS-CDMA," IEEE J. Sel. Areas Commun., vol. 14, no. 8, pp. 1510-1521, Oct. 1996.

[6] A. M. Monk, M. Davis, and L. B. Milstein, "A noise-whitening approach to multiple access noise rejection-Part I: Theory and background," IEEE J. Sel. Areas Commun., vol. 12, no. 5, pp. 817-827, Jun. 1994.

[7] M. Davis, A. Monk, and L. B. Milstein, "A noise-whitening approach to multiple access noise rejection-Part II: Implementation issues," IEEE J. Sel. Areas Commun., vol. 14, no. 8, pp. 1488-1499, Oct. 1996.

[8] Y. Huang and T. S. Ng, "Performance of coherent receiver with weighted despreading sequence for DS-CDMA," Electron. Lett., vol. 33, no. 1, pp. 23-25, Jan. 1997.
[9] — , "DS-CDMA with power control error using weighted despreading sequences over a multi-path Rayleigh fading channel," IEEE Trans. Veh. Technol., vol. 48, no. 4, pp. 1067-1079, Jul. 1999.

[10] — "A DS-CDMA system using despreading sequences weighted by adjustable chip waveforms," IEEE Trans. Commun., vol. 47, no. 12, pp. 1884-1896, Dec. 1999.

[11] - "Capacity enhancement of band-limited DS-CDMA system using weighted despreading function," IEEE Trans. Commun., vol. 47, no. 8, pp. 1218-1226, Aug. 1999.

[12] J. E. Salt and S. Kumar, "Effects of filtering on the performance of QPSK and MSK modulation in D-S spread spectrum systems using RAKE receivers," IEEE J. Sel. Areas Commun., vol. 12, no. 4, pp. 707-715, May 1994.

[13] R. L. Peterson, R. E. Ziemer, and D. E. Borth, Introduction to Spread Spectrum Communications. Upper Saddle River, NJ: PrenticeHall, 1995.

[14] A. Polydoros and C. L. Weber, "Analysis and optimisation of correlative code-tracking loops in spread-spectrum systems," IEEE Trans. Commun., vol. COM-33, no. 1, pp. 30-43, Jan. 1985.

[15] R. D. Gaudenzi and M. Luise, "Decision-directed coherent delay-lock tracking loop for DS-spread-spectrum signals," IEEE Trans. Commun., vol. 39, no. 5, pp. 758-765, May 1991.

[16] J. Viterbi, "Very low rate convolutional codes for maximum theoretical performance of spread-spectrum multiple access channels," IEEE J. Sel. Areas Commun., vol. 8, no. 4, pp. 641-649, May 1990.

[17] L. Yu and J. E. Salt, "A hybrid spreading/despreading function with good SNR performance for band-limited DS-CDMA," IEEE J. Sel. Areas Commun., vol. 14, no. 8, pp. 1576-1582, Oct. 1996.

[18] R. D. Gaudenzi, M. Luise, and R. Viola, "A digital chip timing recovery loop for band-limited CDMA signals," IEEE Trans. Commun., vol. 41, no. 11, pp. 1760-1769, Nov. 1993.

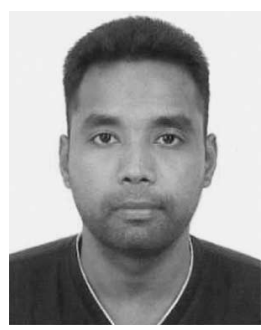

Subramaniam Thayaparan was born in Sri Lanka. $\mathrm{He}$ received the B.Sc. degree from the University of Peradeniya, Peradeniya, Sri Lanka, in 1994 and the Ph.D. degree from the University of Hong Kong, Pokfulam Road, Hong Kong, in 2000, both in electrical and electronic engineering. His dissertation was on the delay-locked loop techniques in directsequence spread-spectrum (SS) receivers.

He worked as an Engineer in Sri Lanka Telecom from July 1995 to January 1996 . He is currently a Senior Integrated Circuit (IC) Design Engineer with Agilent Technologies, Singapore. His current research interests include delaylocked tracking and multiple-access interference reduction techniques in SS systems and Intellectual Property development for communication ICs.

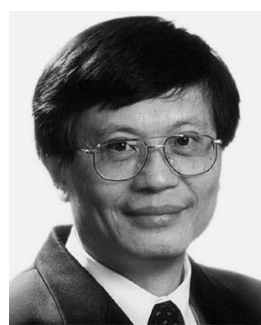

Tung-Sang Ng (S'74-M'78-SM'90-F'03) received the B.Sc. (Eng.) degree from the University of Hong Kong, Pokfulam Road, Hong Kong, in 1972, and the M.Eng.Sc. and Ph.D. degrees from the University of Newcastle, Newcastle, N.S.W., Australia, in 1974 and 1977, respectively, all in electrical engineering.

He was with BHP Steel International and the University of Wollongong, Wollongong, N.S.W., Australia, for 14 years before returning to the University of Hong Kong in 1991, taking up the position of Professor and Chair of Electronic Engineering. He was Head of Department of Electrical and Electronic Engineering from 2000 to 2003 and is currently Dean of Engineering. He has published over 250 international journal and conference papers. His current research interests include wireless communication systems, spread-spectrum techniques, codedivision multiple-access, and digital signal processing.

Dr. Ng is a Fellow of the Institution of Electrical Engineers (IEE) and the Hong Kong Institution of Engineers. He was the General Chair of ISCAS'97 and the VP-Region 10 of the IEEE Circuits and Systems Society in 1999 and 2000. He was an Executive Committee Member and a Board Member of the IEE Informatics Divisional Board (1999-2001) and was an Ordinary Member of the IEE Council (1999-2001). He was awarded the Honorary Doctor of Engineering Degree by the University of Newcastle in 1997, the Senior Croucher Foundation Fellowship in 1999, the IEEE Third Millenium Medal in 2000, and the Outstanding Researcher Award by the University of Hong Kong in 2003. 


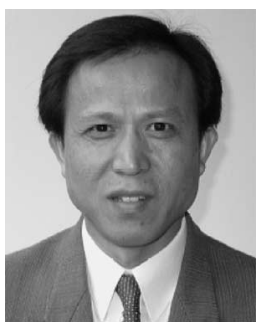

Jiangzhou Wang (M'91-SM'94) received the B.S. and M.S. degrees from Xidian University, Xian, China, in 1983 and 1985, respectively, and the Ph.D. degree (with Greatest Distinction) from the University of Ghent, Gent, Belgium, in 1990, all in electrical engineering.

$\mathrm{He}$ is currently a Professor and Chair with the Department of Electronics, University of Kent, Kent, U.K. From 1995 to 2005 , he was with the University of Hong Kong, where he is still serving as an Honorary Professor. From 1992 to 1995, he was a Senior System Engineer at Rockwell International Corporation (now Conexant). From 1990 to 1992, he was a Postdoctoral Fellow with the University of California at San Diego, La Jolla. He has held a Visiting Professor position with NTT DoCoMo, Tokyo, Japan. He has published over 140 papers, including more than 40 IEEE Transactions/Journal papers in the areas of wireless mobile and spread-spectrum communications. He has written/edited two books, entitled Broadband Wireless Communications (Boston, MA: Kluwer, 2001) and Advances in $3 G$ Enhanced Technologies for Wireless Communications (Norwood, MA: Artech House, 2002), respectively. The latter book has been translated into Chinese. He is also the holder of one U.S. patent for the GSM system.

Dr. Wang is an Editor of the IEEE TRANSACTIONS ON COMMUNICATIONS and a three-time Guest Editor of the IEEE JOURNAL ON SELECTED AREAS IN COMMUNICATIONS (Wideband CDMA in August 2000 and January 2001 and Advances in Multicarrier CDMA in July 2006). He was a Technical Chairman of the IEEE Workshop on 3G Mobile Communications in December 2000. He has been a Technical Committee Member and Session Chair of a number of international conferences. He is listed in Who's Who in the World. 